ESET Secure Authentication ist auf iPhone, Android, BlackBerry, Windows Phone 7 und 8, Windows Mobile und J2ME-basierten Handys lauffähig. Die Lösung unterstützt auch die Authentifizierung basierend auf SMS-Nachrichten. So kann das System auf älteren Mobiltelefonen eingesetzt werden, die von der Anwendung selbst nicht unterstützt werden.

ESET Secure Authentication stellt eine ideale Ergänzung zu den anderen ESET-Lösungen zum Schutz von Unternehmensdaten dar. Während ESET Mobile Security die gespeicherten Daten auf Smartphones vor Malware und Missbrauch schützt, bietet ESA den sicheren Zugriff auf Unternehmensnetzwerke.

Weitere Informationen erhalten Sie unter www.eset.de

\section{E-Mail bleibt Sicherheitsschwachstelle in den Unternehmen}

Deutschland sicher im Netz e. V. (DsiN) veröffentlichte am 08.05.2013 die 3. Studie zur IT-Sicherheitslage im Mittelstand: Während sich das Bewusstsein für IT-Sicherheit in den mittelständischen Unternehmen in Deutschland insgesamt positiv entwickelt und nahezu alle Betriebe inzwischen für einen hinreichenden Internetschutz sorgen, besteht bei der Absicherung der E-Mail-Kommunikation weiterhin Nachholbedarf.

Die Nutzung von E-Mail und Internet ist demnach im vergangenen Jahr weiter gestiegen. Nahezu alle befragten Unternehmen verwenden beide Medien heute für die Abwicklung ihrer Geschäfte. Dabei kommt die E-Mail auf einen Durchdringungsgrad von 97 Prozent, das Internet sogar auf 98 Prozent. Erfreuliche 99 Prozent davon sichern ihren Internetzugang sowie ihre Daten, das Einspielen von Sicherheits-Updates nehmen 97 Prozent der Unternehmen ernst. Obwohl auch die E-Mail als Kommunikationsmedium aus dem geschäftlichen Alltag nicht mehr wegzudenken ist, ergreifen allerdings lediglich 44 Prozent der Befragten Maßnahmen zum Schutz ihrer Informationen auf dem Weg zum Empfänger. Mit einer Verschlechterung um zwei Prozent gegen über dem Vorjahr ist dies der einzige Wert in der Befragung, der sich negativ entwickelt hat.

Mobilnutzung und Cloud sind Handlungsfelder der Zukunft Daneben zeichnen sich zwei weitere Felder ab, in denen Handlungsbedarf besteht. So hat die Nutzung von Smartphones, TabletPCs und Netbooks um 14 Prozentpunkte zugenommen. Die Absicherung der mobilen Geräte stagniert dagegen mit 82 Prozent auf einem verbesserungsbedürftigen Niveau. Neu in die Abfrage integriert wurde im vergangenen Jahr der Themenbereich Cloud Computing. Bereits 17 Prozent befragten Unternehmen arbeiten heute mit Cloud-Anwendungen. Allerdings sind nur einem knappen Drittel dieser Cloud-Nutzer (32 Prozent) die Sicherheitsanforderungen und rechtlichen Rahmenbedingungen dafür umfänglich geläufig, während 27 Prozent sie überhaupt nicht kennen.
Die ausführliche Studie „IT-Sicherheitslage im Mittelstand 2013“ steht auf den DsiN-Internetseiten und auf $h t t p s: / / w w w . d s i n-b l o g . d e$ zum Download bereit. Basis der Erhebung sind die Ergebnisse des „DsiN-Sicherheitschecks", mit dem sich kleine und mittelständische Unternehmen kostenfrei im Internet über den Stand ihrer Informationssicherheit informieren können. Entsprechend der individuellen Ergebnisse liefert der Check produktneutrale und herstellerübergreifende Handlungsempfehlungen, um die Einhaltung von Datenschutz- und Datensicherheitsregeln zu verbessern.

Konzipiert wurde der Sicherheitscheck vom DsiN-Mitglied DATEV mit Unterstützung weiterer Vereinsmitglieder wie BITKOM, SAP und Sophos. Seit 2010 ist er unter https://www.sicher-im-netz. de/unternehmen/DsiN-Sicherheitscheck.aspx verfügbar. Die anonymen Befragungsergebnisse wertet DsiN gemeinsam mit DATEV seit 2011 jährlich in Form der Sicherheitsstudie aus. Für das Jahr 2012 konnte dabei auf eine Basis von mehr als 1.500 durchgeführten Sicherheitschecks zurückgegriffen werden.

Studie „IT-Sicherheitslage im Mittelstand 2013": https://www.sicher-im-netz.de/unternehmen/sicherheitslage_mittelstand_2013. aspx

\section{nPA jetzt auch für ELSTER verwendbar}

„Für Registrierung im ElsterOnline-Portal kann ab sofort auch der neue Personalausweis verwendet werden. Die Identität des Anwenders wird direkt anhand des neuen Personalausweises überprüft. Es muss kein Aktivierungsbrief mehr verschickt werden. Dadurch wird das Verfahren erheblich beschleunigt und vereinfacht." erklärte der IT-Beauftragte der Bayerischen Staatsregierung, Finanzstaatssekretär Franz Josef Pschierer am 17.4.2013. Das ElsterOnline-Portal (www.elsteronline.de) ermöglicht es Bürgerinnen und Bürgern, Steuererklärungen online im Internet auszufüllen und abzugeben. Ferner können diverse Dienste in Anspruch genommen werden, wie zum Beispiel der elektronische Einspruch oder die Auskunft über die Elektronischen Lohnsteuerabzugsmerkmale. Das ElsterOnline-Portal erzeugt ein elektronisches Zertifikat, das zur sicheren Authentifizierung elektronischer Steuererklärungen genutzt werden kann. Der Bürger kann, so Pschierer, bei Beantragung des neuen Personalausweises entscheiden, ob er die Signaturanwendung, die sogenannte eiD-Funktion, freischaltet oder nicht. Von den 2,5 Millionen neuen Ausweisen in Bayern haben rund 30 Prozent, das sind 700.000, eine eingeschaltete eiD-Funktion. „Bayern hat damit in Deutschland eine Spitzenposition inne. Der Bürger hat im Jahr rund 1,8 Behördenkontakte. Dabei eingerechnet ist die jährliche Abgabe der Steuererklärung. Die Nutzung von ELSTER durch die eiD des Personalausweises wird dem sicheren eGovernment einen weiteren Schub geben", so Pschierer abschließend. 